\title{
Tibial Nerve
}

National Cancer Institute

\section{Source}

National Cancer Institute. Tibial Nerve. NCI Thesaurus. Code C52809.

One of two major branches of the sciatic nerve. It innervates the muscles of the posterior aspect of the lower limb, the muscles of the plantar aspect of the foot, as well as the skin on the posterior aspect of the leg and on the sole of the foot. 